Applied Mathematical Sciences, Vol. 9, 2015, no. 20, 967 - 971

HIKARI Ltd, www.m-hikari.com

http://dx.doi.org/10.12988/ams.2015.4121045

\title{
Restrained Weakly Connected Independent Domination in the Join of Graphs
}

\author{
Rene E. Leonida \\ Mathematics Department \\ College of Natural Sciences and Mathematics \\ Mindanao State University \\ Fatima, General Santos City, Philippines
}

Copyright (c) 2015 Rene E. Leonida. This is an open access article distributed under the Creative Commons Attribution License, which permits unrestricted use, distribution, and reproduction in any medium, provided the original work is properly cited.

\begin{abstract}
In this paper, we explore the concept of restrained weakly connected independent domination in graphs. In particular, we characterized the restrained weakly connected independent dominating sets in the join of graphs and obtain the restrained weakly connected independent domination numbers. A connected graph is constructed with a given weakly connected independent domination number, restrained weakly connected independent domination number, and maximum weakly connected independent domination number.
\end{abstract}

Mathematics Subject Classification: 05C69

Keywords: domination, restrained domination, weakly connected domination, independent domination, restrained weakly connected independent domination

\section{Introduction and Preliminary Results}

Let $G=(V(G), E(G))$ be a simple connected graph. For any vertex $v \in V(G)$, the open neighborhood of $v$ is the set $N(v)=\{u \in V(G): u v \in$ 
$E(G)\}$ and the closed neighborhood of $v$ is the set $N[v]=N(v) \cup\{v\}$. For a set $X \subseteq V(G)$, the open neighborhood of $X$ is $N(X)=\bigcup_{v \in X} N(v)$ and the closed neighborhood of $X$ is $N[X]=X \cup N(X)$. A subset $S$ of $V(G)$ is an independent set if for every $x, y \in S, x y \notin E(G)$. The independence number $\beta(G)$ of $G$ is the largest cardinality of an independent set of $G$. A subset $S$ of $V(G)$ is called weakly connected if the subgraph $\langle S\rangle_{w}=\left(N_{G}[S], E_{W}\right)$ weakly induced by $S$, is connected, where $E_{W}$ is the set of all edges with at least one vertex in $S$.

A subset $S$ of $V(G)$ is a dominating set of $G$ if for every $v \in V(G) \backslash S$, there exists $u \in S$ such that $u v \in E(G)$. The domination number of $G$, denoted by $\gamma(G)$, is the smallest cardinality of a dominating set of $G$. A dominating set of $G$ which is independent (resp., weakly connected) is called an independent (resp., weakly connected) dominating set of $G$. The independent (resp. weakly connected) domination number of $G$, denoted by $i(G)\left(\operatorname{resp} ., \gamma_{w}(G)\right)$, is the smallest cardinality of an independent (resp., weakly connected) dominating set of $G$. An independent dominating set of $G$ which is weakly connected is called a weakly connected independent dominating set of $G$. The weakly connected independent domination number of $G$, denoted by $i_{w}(G)$, is the smallest cardinality of a weakly connected independent dominating set of $G$. Similarly, the upper weakly connected independent domination number of $G$, denoted by $\beta_{w}(G)$, is the largest cardinality of a weakly connected independent dominating set of $G$.

A dominating set $S$ is called a restrained dominating set of $G$ if for every $u \in V(G) \backslash S$, there exists $w \in V(G) \backslash S$ such that $u w \in E(G)$. The restrained domination number of $G$, denoted by $\gamma_{r}(G)$, is the smallest cardinality of a secure dominating set of $G$. A set $S$ is called a restrained weakly connected independent dominating set of $G$ if $S$ is a weakly connected independent dominating set of $G$ and for every $u \in V(G) \backslash S$, there exists $w \in V(G) \backslash S$ such that $u w \in E(G)$. The restrained weakly connected independent domination number of $G$, denoted by $i_{r w}(G)$, is the smallest cardinality of a restrained weakly connected dominating set of $G$.

The concept of weakly connected independent domination is discussed in [2] [3, and [4]. Another domination parameter is the restrained domination which was discussed in [1] and [5]. A combination of these two concepts give rise to a new variant of domination called restrained weakly connected independent domination.

Remark 1.1 Let $G$ be a graph of order $n$. Then $1 \leq \gamma(G) \leq i_{w}(G) \leq i_{r w}(G)$.

Theorem 1.2 Let $a, b$, and $c$ be positive integers such that $1 \leq a \leq b$ and $c=a+b-1$. Then there exists a connected graph $G$ such that $i_{w}(G)=a$, $i_{r w}(G)=b$ and $\beta_{w}(G)=c$. 
Proof: Consider the path $P=\left[u_{1}, v_{1}, u_{2}, v_{2} \ldots, u_{2 a-2}, v_{2 a-2}, u_{2 a-1}, u_{2 a}\right]$. Let $G$ be a graph obtained from $P$ by adding the edges $u_{i} u_{i+1}$ for $i=1,2, \ldots, a+1$ and adding the edges $u_{1} w_{j}$, for $j=0,1,2, \ldots, b-a$ (see Figure 1). Then

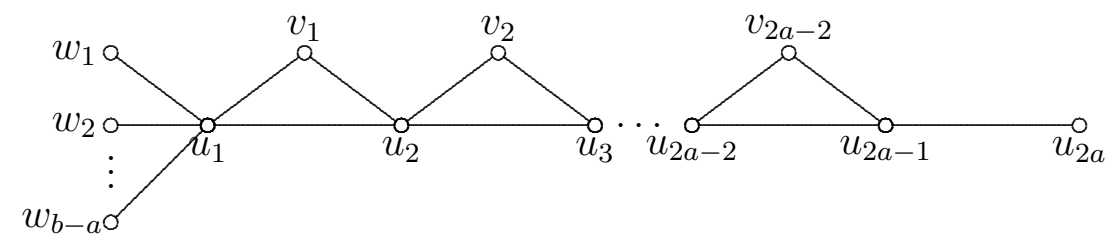

Figure 1: A graph $G$ with $i_{w}(G) \leq i_{r w}(G)$

$\left\{u_{1}, u_{3}, \ldots, u_{2 a-1}\right\}$ is a weakly connected independent dominating set of $G$, $\left\{u_{2}, u_{4}, \ldots, u_{2 a}\right\} \cup\left\{w_{1}, w_{2}, \ldots, w_{b-a}\right\}$ is a restrained weakly connected independent dominating set of $G$, and $\left\{w_{1}, w_{2}, \ldots, w_{b-a}\right\} \cup\left\{v_{1}, v_{2}, \ldots, v_{2 a-1}, u_{2 a}\right\}$ is a maximum weakly connected independent dominating set of $G$. Hence, $i_{w}(G)=a, i_{r w}(G)=$ $b$ and $\beta_{w}(G)=c$.

Corollary 1.3 The difference $i_{r w}-i_{w}$ can be made arbitrarily large.

\section{Results}

The join of two graphs $G$ and $H$, denoted by $G+H$, is the graph with vertex-set $V(G+H)=V(G) \cup V(H)$ and edge-set $E(G+H)=E(G) \cup E(H) \cup\{u v$ : $u \in V(G), v \in V(H)\}$.

Theorem 2.1 Let $G$ be a graph of order $n \geq 2$. Then $i_{r w}\left(K_{1}+G\right)=1$ if and only if $G$ has no isolated vertex.

Proof: Suppose $i_{r w}\left(K_{1}+G\right)=1$. Let $S=\{v\}$ be a restrained weakly connected independent dominating set of $K_{1}+G$. Suppose $v \in V\left(K_{1}\right)$. Since $S$ is a restrained dominating set, for each $x \in V\left(K_{1}+G\right) \backslash S=V(G)$, there exists $y \in V(G)$ such that $x y \in E(G)$. Hence, $G$ has no isolated vertex. Suppose $v \in V(G)$. Since $S$ is a dominating set, for each $u \in V(G) \backslash\{v\}, u v \in E(G)$. Therefore, $G$ has no isolated vertex.

Conversely, suppose $G$ has no isolated vertex. Let $K_{1}=\langle\{v\}\rangle$ and set $S=\{v\}$. Then $S$ is a weakly connected independent dominating set of $K_{1}+G$. Let $u \in V\left(K_{1}+G\right) \backslash S$. Then $u \in V(G)$. Thus, $u \in V(\langle C\rangle)$, where $C$ is a nontrivial component of $G$. Hence, there exists $w \in V(\langle C\rangle) \subseteq V\left(K_{1}+G\right) \backslash S$ such that $u w \in E\left(K_{1}+G\right)$. Therefore, $S$ is a restrained weakly connected independent dominating set of $K_{1}+G$. 
Theorem 2.2 Let $G$ be a graph of order $n \geq 3$. Then $i_{r w}\left(K_{1}+G\right)=2$ if and only if $G=\langle C\rangle \cup\langle\{v\}\rangle$, where $C$ is a nontrivial component of $G$ with $\gamma(\langle C\rangle)=1$.

Proof: Suppose $i_{r w}\left(K_{1}+G\right)=2$. Let $S=\{u, v\}$ be a restrained weakly connected independent dominating set of $K_{1}+G$. Since $S$ is independent, $S \subseteq V(G)$ and $u v \notin E(G)$. Since $i_{r w}\left(K_{1}+G\right)=1, G$ must have an isolated vertex by Theorem 2.1. But $u$ and $v$ are not both isolated vertices since $S$ is a restrained dominating set. Hence, $G$ has one isolated vertex, say $v$. The vertex $u$ is a dominating vertex of the other component of $G$. Hence, $G=\langle C\rangle \cup\langle\{v\}\rangle$, where $C$ is a nontrivial component of $G$ with $\gamma(\langle C\rangle)=1$.

Suppose $G=\langle C\rangle \cup\langle\{v\}\rangle$, where $C$ is a nontrivial component of $G$ with $\gamma(\langle C\rangle)=1$. Then $i_{r w}\left(K_{1}+G\right) \neq 1$. Let $S=\{v, w\}$, where $w$ is a dominating vertex of $\langle C\rangle$. Clearly $S$ is a restrained weakly connected independent dominating set of $K_{1}+G$. Therefore, $i_{r w}\left(K_{1}+G\right)=2$.

The next theorem can be found in [2].

Theorem 2.3 Let $G$ and $H$ be graphs. Then $S \subseteq V(G+H)$ is a weakly connected independent dominating set of $G+H$ if and only if either $S$ is an independent dominating set of $G$ or $S$ is an independent dominating set of $H$.

A similar result characterizes the restrained weakly connected independent dominating set of $G+H$.

Theorem 2.4 Let $G$ and $H$ be nontrivial and nonempty graphs. Then $S \subseteq$ $V(G+H)$ is a restrained weakly connected independent dominating set of $G+H$ if and only if $S$ is an independent dominating set of $G$ or $S$ is a independent dominating set of $H$.

Proof: If $S \subseteq V(G+H)$ is a restrained weakly connected independent dominating set of $G+H$, then $S$ is an independent dominating set of $G$ or $S$ is a independent dominating set of $H$ by Theorem 2.3 .

Conversely, suppose $S$ is an independent dominating set of $G$. By Theorem 2.3, $S$ is a weakly connected independent dominating set of $G+H$. Let $x \in V(G+H) \backslash S$. If $x \in V(G) \backslash S$, then $x y \in E(G+H)$ for all $y \in$ $V(H) \subseteq V(G+H) \backslash S$. Suppose $x \in V(H)$. Since $G$ is nontrivial, there exists $z \in V(G) \backslash S \subseteq V(G+H) \backslash S$ such that $x z \in E(G+H)$. Hence, $S$ is a restrained weakly connected independent dominating set of $G+H$. Similarly, if $S$ is a independent dominating set of $H$, then $S$ is a restrained weakly connected independent dominating set of $G+H$.

Corollary 2.5 Let $G$ and $H$ be nontrivial and nonempty graphs. Then 


$$
i_{r w}(G+H)=\min \{i(G), i(H)\} .
$$

Proof: Assume that $i(G) \leq i(H)$. Let $S$ be a minimum restrained weakly connected independent dominating set of $G+H$. Suppose that $S$ is not a minimum independent dominating set of $G$. Then there exists an independent dominating set $S^{*}$ of $G$ such that $\left|S^{*}\right|<|S|$. By Theorem 2.4, $S^{*}$ is a restrained weakly connected independent dominating set of $G+H$. This contradicts the fact that $S$ is a minimum restrained weakly connected independent dominating set of $G+H$. Thus, $S$ is a minimum independent dominating set of $G$. Hence, $i_{r w}(G+H)=|S|=i(G)$. Therefore, $i_{r w}(G+H)=\min \{i(G), i(H)\}$.

Theorem 2.6 Let $G$ be a graph of order $m \geq 3$ and let $n \geq 3$ be an integer. If $G$ has no isolated vertex, then

$$
i_{r w}\left(G+\overline{K_{n}}\right)=\min \{i(G), n\} .
$$

Proof: Let $S$ be a minimum restrained weakly connected independent dominating set of $G+\overline{K_{n}}$. Then either $S \subseteq V(G)$ or $S \subseteq V\left(\overline{K_{n}}\right)$ since $S$ is independent. Thus either $S$ is a minimum independent dominating set of $G$ or $S=V\left(\overline{K_{n}}\right)$. Hence, $i_{r w}\left(G+\overline{K_{n}}\right)=\min \{i(G), n\}$.

\section{References}

[1] S. R. Canoy, Jr., Restrained Domination in Graphs Under Some Binary Operations, Applied Mathematical Sciences, 8(2014), 6025-6031. http://dx.doi.org/10.12988/ams.2014.48597

[2] R. E. Leonida, Weakly Connected Independent Dominations in the Join of Graphs, International Math. Forum, 8(2013), 1767-1771. http://dx.doi.org/10.12988/imf.2013.39170

[3] R. E. Leonida and S. R. Canoy, Jr., Weakly Convex and Weakly Connected Independent Dominations in the Corona of Graphs, International Mathematical Forum, 8(2013), 1515-1522. http://dx.doi.org/10.12988/imf.2013.37131

[4] R.E. Leonida, E.P. Sandueta, and S.R. Canoy, Jr., Weakly Connected Independent and Weakly Connected Total Dominations in a Product of Graphs, Applied Mathematical Sciences, 8(2014), 5743-5749. http://dx.doi.org/10.12988/ams.2014.47587

[5] N. Tuan and S.R. Canoy, Jr. Independent Restrained Domination in Graphs, Applied Mathematical Sciences, 8(2014), 6033-6038. http://dx.doi.org/10.12988/ams.2014.48598

\section{Received: January 3, 2015; Published: February 1, 2015}

